\title{
Translational and Rotational Diffusion in Water in the Gigapascal Range
}

\author{
L. E. Bove, ${ }^{1,2}$ S. Klotz, ${ }^{1}$ Th. Strässle, ${ }^{3}$ M. Koza, ${ }^{4}$ J. Teixeira, ${ }^{5}$ and A. M. Saitta ${ }^{1}$ \\ ${ }^{1}$ IMPMC, CNRS-UMR 7590, Université Pierre \& Marie Curie, 75252 Paris, France \\ ${ }^{2}$ Ecole Polytech Fed Lausanne, Inst Condensed Matter Phys, EPSL, CH-1015 Lausanne, Switzerland \\ ${ }^{3}$ Paul Scherrer Institut, CH-5232 Villigen PSI, Switzerland \\ ${ }^{4}$ Institut Laue Langevin, BP 156, F-38042 Grenoble, France \\ ${ }^{5}$ Laboratoire Léon Brillouin (CEA/CNRS), CEA Saclay, F-91161 Gif-sur-Yvette, France
}

(Received 11 March 2013; published 28 October 2013)

\begin{abstract}
First measurements of the self-dynamics of liquid water in the GPa range are reported. The GPa range has here become accessible through a new setup for the Paris-Edinburgh press specially conceived for quasielastic neutron scattering studies. A direct measurement of both the translational and rotational diffusion coefficients of water along the $400 \mathrm{~K}$ isotherm up to $3 \mathrm{GPa}$, corresponding to the melting point of ice VII, is provided and compared with molecular dynamics simulations. The translational diffusion is observed to strongly decrease with pressure, though its variation slows down for pressures higher than $1 \mathrm{GPa}$ and decouples from that of the shear viscosity. The rotational diffusion turns out to be insensitive to pressure. Through comparison with structural data and molecular dynamics simulations, we show that this is a consequence of the rigidity of the first neighbors shell and of the invariance of the number of hydrogen bonds of a water molecule under high pressure. These results show the inadequacy of the Stokes-EinsteinDebye equations to predict the self-diffusive behavior of water at high temperature and high pressure, and challenge the usual description of hot dense water behaving as a simple liquid.
\end{abstract}

DOI: 10.1103/PhysRevLett.111.185901

PACS numbers: 66.10.C-, 45.20.dc, 62.50.-p, 78.70.Nx

Water is, by far, the most well-known substance in nature, as essentially most of its properties have been measured with high accuracy [1], at least at atmospheric or moderate pressure. Water under high pressure (HP) has also been the subject of several investigations [2-13], as it affects fields ranging from condensed matter physics to planetary science. However, while structural [2] and vibrational properties [3] of water have been explored up to several GPa, the knowledge of water self-dynamics under high pressure lags far behind [14-17]. Information on water diffusion under HP conditions is of paramount importance for several issues in applied and fundamental science. As examples, the diffusion of water at pressures of few GPa, typical of the transition zone of the Earth's mantle, has a strong incidence on the processes governing intermediate-depth seismicity. New phases of water characterized by peculiar diffusive behavior, as free rotation of water molecules [12] (plastic phases), or proton free diffusion [13] (superionic phase) are predicted by computer simulations [2-4] at pressures of several GPa. Their characterization is essential in order to develop reliable models of planetary interiors.

We developed a new setup for the Paris-Edinburgh (PE) press [18,19] adapted to quasielastic incoherent neutron scattering (QENS) measurements [20], giving access to hydrogen diffusion and water molecular reorientation in the $\mathrm{GPa}$ range (up to $5 \mathrm{GPa}$ and $550 \mathrm{~K}$ ). Incoherent neutron scattering is a unique probe of individual motions of atoms and, in particular, of hydrogen, due to its huge incoherent cross section, typically 2 orders of magnitude larger than in other elements. Applied to water, QENS measures the time-dependent correlation function of hydrogen, providing information on the molecular diffusion and the motion of hydrogen atoms around the molecular center of mass. At high temperature, this motion coincides with the molecule reorientation. Consequently, QENS offers the unique possibility of analyzing both atomic and molecular motions [20,21] on the picosecond time scale and on the length scale of atomic distances. The necessity for QENS measurements of large samples for energy analysis, had so far prevented the use of this technique at pressures higher than few kbars [14]. Earlier information on water self-dynamics under HP was derived from NMR measurements and limited to pressures lower than $1 \mathrm{GPa}[15,16]$.

We have studied the dynamics of liquid water at $400 \mathrm{~K}$ up to $3 \mathrm{GPa}$ (Fig. 1). The large density variation (40\%) allows for probing the interplay between molecular reorientation, diffusion, and structural modifications of the hydrogen bonded (HB) network [22-26], in a regime where water is non anomalous [22] and shows large fluidity [9]. The highest pressure (3 GPa) reached is the crystallization point of ice VII. "Plastic ice VII" [12], i.e., a crystalline phase where water molecules freely rotate around their center of mass, is predicted to exist along the melting line of ice VII. Further data were collected increasing pressure and warming along the melting line up to $430 \mathrm{~K}$ and $3.3 \mathrm{GPa}$ with $T$ steps of $10 \mathrm{~K}$.

In Fig. 2, we show a few spectra collected at $1.8 \mathrm{GPa}$, as a function of the exchanged energy, $E$, for selected momentum transfers, $Q$. The instrumental resolution function, 


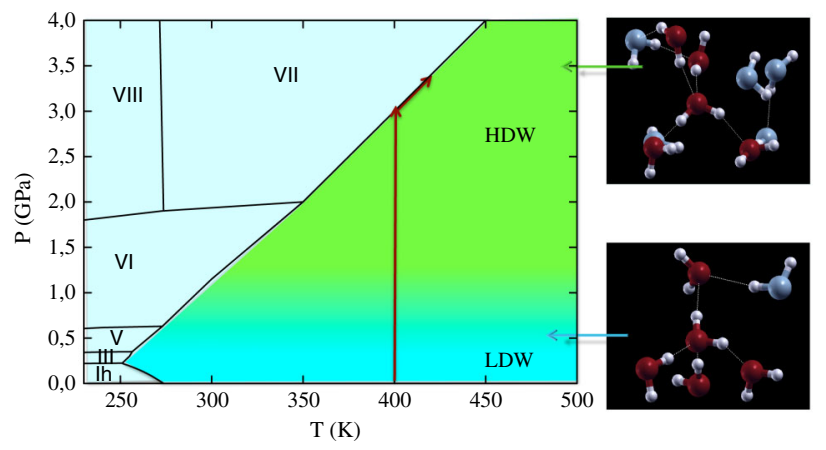

FIG. 1 (color online). Phase diagram of water in the $P-T$ range considered, extended from MD results of [5]. The blue-green color boundary indicates the collapse of the second coordination shell on the first one (see also Supplemental Material subsection [27]). Arrows show the thermodynamic path followed in the present experiment. The two simulations snapshots on the left indicate the first neighbors (oxygens in red) and the second neighbors (oxygens in light-blue) of a central water molecule at ambient pressure (lower panel) and at high pressure (upper panel).

as measured from a vanadium scan, is also reported for comparison. The details of the data reduction are described in the Supplemental Material [27].

To fit the experimental spectra, we assumed a decoupling between vibrational, translational, and rotational motions $[20,21]$, which is essential to obtain an analytical expression for the dynamic structure factor. The vibrational dynamics include an elastic component described by a $Q$-dependent factor and several inelastic components. In practice, this component introduces a small flat background that can be barely appreciated in the raw data and an overall scaling factor. The approximation of decoupling the translational

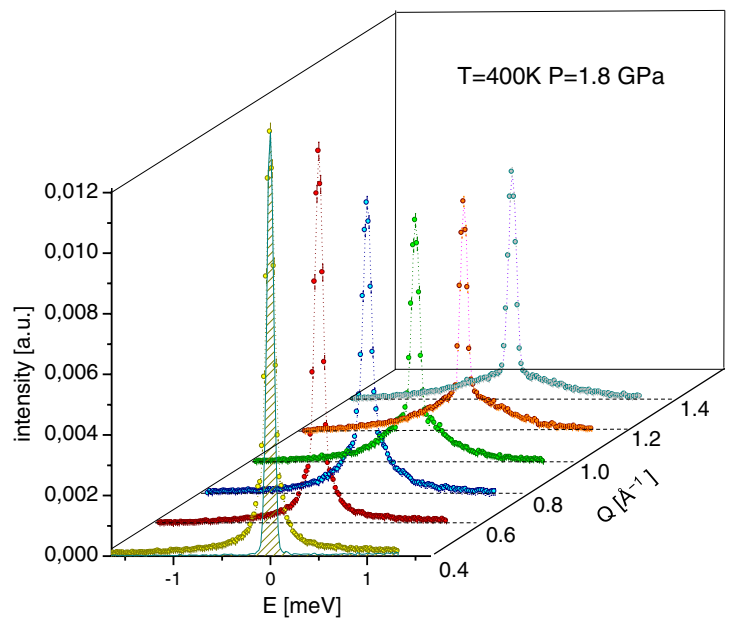

FIG. 2 (color online). Example of $12 \mathrm{~h}$ scan collected on water at $400 \mathrm{~K}$ and $1.8 \mathrm{GPa}$ in the HP-QENS setup of the PE press, on IN6 at ILL. Different colors represent different $Q$ cuts from 0.4 to $1.4 \mathrm{~A}^{-1}$. The shadowed region represents the resolution as measured by a vanadium scan. and rotational motions has been shown to fail at low temperatures where water molecule rotation and structural relaxation are strongly entangled [23,24]. In this regime, MD simulations indicate that QENS data provide little information about water rotation [25] and a consistent interpretation of QENS data in terms of translational jumps has recently been presented [24]. However, at high temperatures, water rotations clearly contribute to the measured dynamics, and the hypothesis of translational-rotational decoupling is reasonable, as the coupling exponentially decreases with temperature [23]. Possible rototraslational coupling effects would eventually affect QENS intensity only at the smaller $Q$ values of the experiment, where the rotational term is less intense [26].

We thus fitted the data with the sum of two Lorentzians, taking into account both translational and rotational motions [24]:

$$
\begin{aligned}
S_{s}(Q, E)= & e^{-\left\langle u^{2}\right\rangle Q^{2} / 3}\left[A(Q) \frac{\Gamma_{T}(Q)}{E^{2}+\Gamma_{T}^{2}(Q)}\right. \\
& \left.+B(Q) \frac{2 D_{R}+\Gamma_{T}(Q)}{E^{2}+\left[2 D_{R}+\Gamma_{T}(Q)\right]^{2}}\right] .
\end{aligned}
$$

The relative amplitudes of the two Lorentzians and their FWHM, i.e., $\Gamma_{T}(Q)$ (translational contribution) and $2 D_{R}+\Gamma_{T}(Q)$ (rotational contribution), are taken to be free parameters. Further details on this choice are given in the Supplemental Material [27].

In Fig. 3, we show an example of fit results compared to the measured intensity at $P=0.6$ and $1.5 \mathrm{GPa}$. The two Lorentzians describing the translational (narrower) and rotational (broader) contributions are readily separable. The parameter $\Gamma_{T}(\mathrm{Q})$ is proportional to the translational diffusion constant $D_{T}$, while the width of the broad

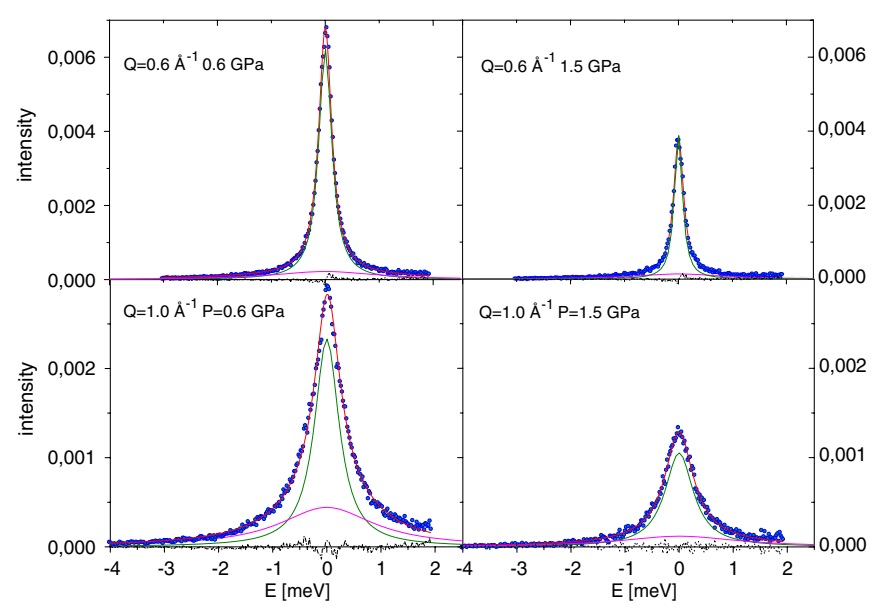

FIG. 3 (color online). Example of fit result (red line) compared to the measured intensity (blue dots) from water at $400 \mathrm{~K}$ at $P=0.6$ and $1.5 \mathrm{GPa}$ for $Q$-selected values. The translational (rotational) contribution is shown in green (magenta). Black dashed curves represent fit residues. 
Lorentzian determines the rotational coefficient $D_{R}$, which, within the error bars, is constant with $Q$, as expected for a localized motion.

The $Q$-dependent translational parameter $\Gamma_{T}(Q)$ is smaller at high momentum transfer than expected from a continuous translational diffusion $\left(D_{T} Q^{2}\right)$ [20,21,24]. In the $Q$ range explored, $\Gamma_{T}(Q)$ can be described as $\Gamma_{T}=$ $D_{T} Q^{2} /\left(1+d^{2} Q^{2} / 6\right)$ with $d$ representing an apparent jump length [21]. The parameter $d$ slightly varies with pressure from a value of $1.65 \AA$ at ambient pressure to $1.45 \AA$ at the higher pressures.

The pressure dependence of the translational $D_{T}$ and of rotational $D_{R}$ diffusion coefficients is reported in Fig. 4. $D_{T}$ is also compared with the macroscopic diffusion coefficient measured by NMR at the same temperature and lower pressures [28]. The agreement is reasonably good

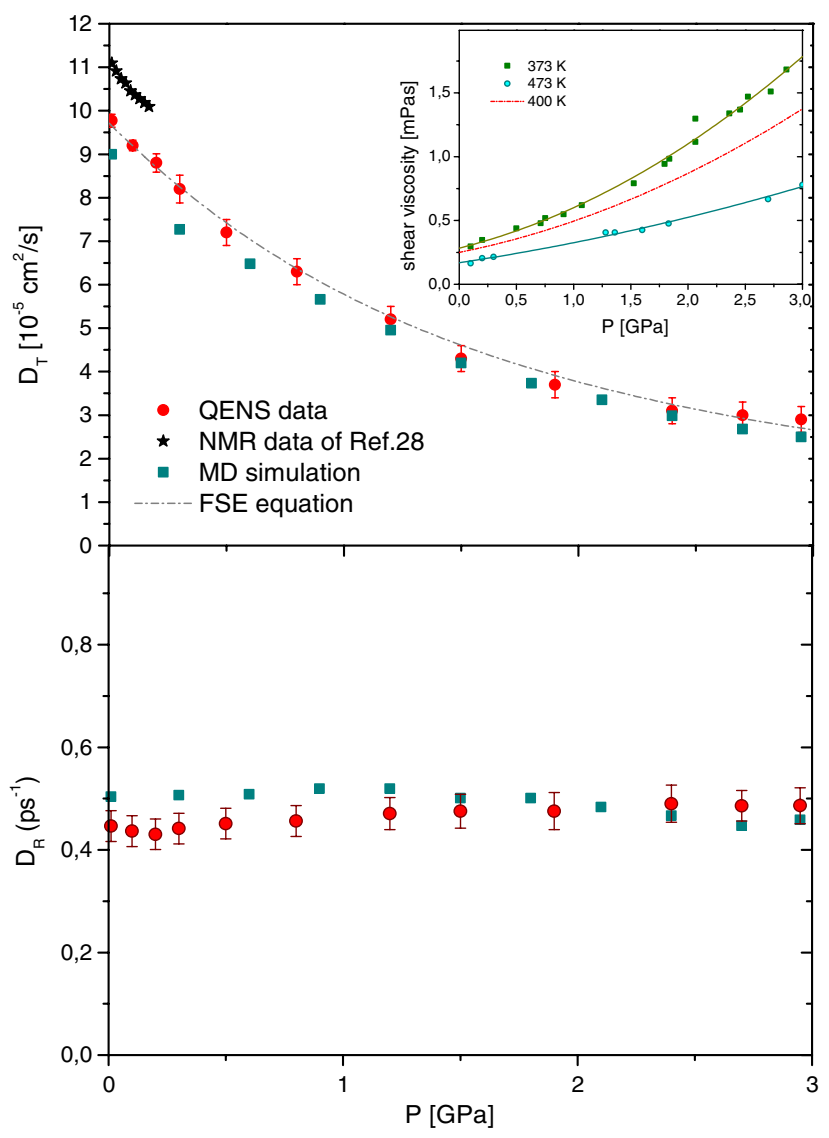

FIG. 4 (color online). Pressure dependence of the translational $D_{T}$ and of rotational $D_{R}$ diffusion coefficients along the isotherm $400 \mathrm{~K}$ as measured by QENS (red dots) and by MD simulations performed by using TIP4P2005 potential for water (blue squares). Black stars represent the available low-pressure data [28] on $D_{T}$ as measured by NMR at $403 \mathrm{~K}$. The gray curve represents the FSE equation [31] prediction with a fractional coefficient $\xi=0.76$. In the inset, we show the shear viscosity dependence on pressure along the $400 \mathrm{~K}$ isotherm (red curve), interpolated from data of Ref. [9] at $373 \mathrm{~K}$ (green dots), and at $437 \mathrm{~K}$ (cyan dots). considering the different techniques. $D_{T}$ shows a marked dependence on pressure in the explored $P$ range, but its variation is almost twice slower than that of the shear viscosity, $\eta$, derived from Ref. [9] (insert Fig. 4). Along the $400 \mathrm{~K}$ isotherm, the product $D_{T} \eta$ varies from $2.4 \times 10^{-8} \mathrm{~Pa} \mathrm{~cm}^{2}$, at $0.1 \mathrm{GPa}$, to $4.0 \times 10^{-8} \mathrm{~Pa} \mathrm{~cm}^{2}$, at $3 \mathrm{GPa}$ (see Table in Supplemental Material [27] for details). This means that the Stokes Einstein (SE) relation, $D_{T} \eta=$ $k T / C \pi a$, which predicts a constant product of diffusion and shear viscosity at constant temperature is violated. Here $C$ is a constant depending of the geometry of the motion [28-30], and $a=1.38 \AA$ is the hydrodynamic radius of the molecule, is violated. Previous NMR [16] and tracer diffusion data [28] on heavy water found that the SE equation in the stick limit $(C=6)$ was accurate within $10 \%$ up to $0.9 \mathrm{GPa}$ at $363 \mathrm{~K}$. The same agreement is observed in our data along the $400 \mathrm{~K}$ isotherm at moderate pressures $(P: 0.1-1 \mathrm{GPa})$, while a stronger deviation from the $\mathrm{SE}$ is observed close to the melting line. The value of $D_{T}$ measured in these conditions $\left(2.95 \times 10^{-5} \mathrm{~cm}^{2} / \mathrm{s}\right)$ is approximately 3 times larger than at the melting line at ambient pressure $\left(1.1 \times 10^{-5} \mathrm{~cm}^{2} / \mathrm{s}\right.$ [28]). This contrasts with the behavior of the shear viscosity, which has almost the same value at these $P / T$ conditions, i.e., $1.2 \mathrm{mPa}$ s $(273 \mathrm{~K}, 1 \mathrm{bar})$ and $1.35 \mathrm{mPa}$ ( $400 \mathrm{~K}, 2.9 \mathrm{GPa}[28])$. Hence, the product $D_{T} \eta / T$ increases by a factor of 2 from 4.8 to $9.8 \times 10^{11} \mathrm{~Pa} \mathrm{~cm}^{2} / \mathrm{K}$, i.e., the $\mathrm{SE}$ relation is again violated. Violation of the SE equation is commonly observed in glass forming liquids when approaching their glass transition temperature (usually for $T<1.3 \mathrm{Tg}$ ) [31]. However, under these conditions, the viscosity of the liquid is extremely high, normally 5-6 orders of magnitude higher than at high temperature (while its density variation is limited to some percents), and the break down of the SE equation is linked to the onset of dynamical heterogeneities in the system [31,32].

Conversely, water at $400 \mathrm{~K}$ shows high fluidity even at the higher investigated pressures, where the viscosity of the system is comparable to that of water at ambient conditions [9], while the density is almost $40 \%$ higher.

The failure of the SE equation observed in hot water under compression seems most likely connected to the free volume reduction and the consequent onset of the hopping phenomenon, which is clearly observed in our data by the saturation of the translational component as a function of $Q$. Simulations of hard sphere (HS) liquids under compression [32] show that the presence of hopping motion is responsible for the observed SE breaking. However, the compressed HS liquid shows dynamical heterogeneities, as its viscosity is increasing by several orders of magnitude. Thus, the effects of density and viscosity variations can not be easily disentangled. The link between free volume reduction, onset of hopping phenomenon, and SE breaking in high fluidity liquids under compression deserves further theoretical investigations. 
As for network forming liquids at low temperature, a fractional SE equation (FSE) well describes the observed trend of translational diffusion with pressure (Fig. 4) with a fractional coefficient $\xi$ of 0.76 that matches the prediction of the entropic barrier hopping theory [31].

Even more striking is the pressure behavior of $D_{R}$, reported in Fig. 4, bottom panel which is almost insensitive to pressure variation, through the wide range probed. This result indicates a clear break down of the Debye equation [30], which establishes a proportional relation between the rotational diffusion coefficient and the inverse of the liquid shear viscosity. First principles molecular dynamics simulations in the 300-900 $\mathrm{K}$ temperatures range and up to pressures of several GPa have recently predicted [7] the existence of such a decoupling in high temperature water under pressure.

A similar decoupling was already observed by NMR measurements under pressure by Jonas and co-workers in liquid water at $363 \mathrm{~K}[15,16]$. The authors measured, though in a limited pressure range (up to $0.9 \mathrm{GPa}$ ), a pressure-independent rotational diffusion time for water. This behavior was attributed to the possible $\mathrm{H}$-bond distortion and breaking under pressure $[15,16]$. The availability nowadays of detailed structural data of water at high temperature in the GPa range [2,6,7] showing the persistence of $\mathrm{H}$ bonds in these conditions, questions this interpretation. The observed decoupling seems though to be a consequence of the different way the HB network modification under pressure affects the translational and rotational motions.

While temperature is more effective in destroying tetrahedricity [5-7] in water, and water above $700 \mathrm{~K}$ shows a simple liquid like structure [6,7], the effect of pressure on water structure is more subtle, as it does not change substantially the number and strength of $\mathrm{H}$ bonds, but the $\mathrm{O}-\mathrm{O}-\mathrm{O}$ angles are modified so that the compressed liquid begins to coordinate water molecules from the out-of-firstshell neighbors tending toward a more compact structure $[4,6]$. Interestingly, the central pentamer, constituted by the central molecule and its four coordinated neighbors (Fig. 1), hardly changes under pressure, even if the overall high-pressure structure develops characteristic simple liquid features, as shown by the $\mathrm{O}-\mathrm{O}$ pair distribution function. As shown in structural studies [6,7], in published [5,33] and new MD simulations (for details, see Supplemental Material [27]), the first shell of neighbors of a water molecule shows a density-independent $\mathrm{O}-\mathrm{O}$ distance for the nearest neighbor oxygen atoms. The radial distribution function of the second coordination shell reveals, instead, a difference between ambient and elevated $P-T$, associated with the gradual transformation towards HDW [4]. Calculations find that, at $400 \mathrm{~K}$, the complete transformation to HDW is achieved at relatively low pressures ( $\sim 0.5 \mathrm{GPa})[5]$, while, under further compression, the second shell radial distribution approximately scales with density. The rotation time associated to hydrogen motions depends mainly on the first shell structural environment and on the number and strength of HB per water molecule, which varies only a few percent in the explored pressure range. Thus, its observed invariance under compression is a consequence of the low dependence of these quantities over pressure.

The observed independence of the rotational diffusion with pressure is also consistent with earlier theoretical predictions $[33,34]$ indicating that the orientational order of water is almost insensitive to pressure along the $400 \mathrm{~K}$ isotherm.

The time scale associated with the observed rotational motions is $\tau_{1}=1 / 2 D_{R}$, as only the first spherical harmonic contributes significantly to the scattered intensity within the $Q$ range covered by the experiment (see Supplemental Material [27]). $\tau_{1}$ is of the order of $1.1 \mathrm{ps,}$ a value comparable with the available low-pressure data of the dipole reorientation time $\tau_{2}$ measured by NMR and extrapolated to $400 \mathrm{~K}, \tau_{2}=0.6$ [5] and $\tau_{2}=0.55 \mathrm{ps}$ [35]. The quantitative agreement between the QENS data and NMR data leads to two conclusions: first, the hydrogen self-motion time scale and the molecular reorientation coincide at high temperature, as QENS becomes sensitive to the same reorientation motion as other techniques probing the time scale of the dipole reorientation. Second, the ratio between the two reorientation times, $\tau_{1}$ and $\tau_{2}$, is closer to 2 , as predicted by an extended jump model [36], rather than 3 , as for a continuous diffusion.

Under further compression, water crystallized in ice VII $(P=3 \mathrm{GPa}, 400 \mathrm{~K})$ and no hint of water rotational dynamics was observed in the crystal and along the crystallization line, up to $P=3.3 \mathrm{GPa}$ and $430 \mathrm{~K}$ : the collected spectra became indistinguishable from the experimental resolution and the observed decreasing in elastic intensity was fully accounted by the Debye Waller factor (vibrational dynamics). This indicates either that the predicted plastic phase of water (12) is located at higher $P / T$ conditions or that water rotation at that temperature occurs on a longer time scale than that probed in the present experiment (of about $10 \mathrm{ps}$ ).

Interestingly, if we compare the diffusion coefficient values measured along the melting line in the liquid phase, $D_{T}=(2.95 \pm 0.2) \times 10^{-5} \mathrm{~cm}^{2} / \mathrm{s}$, with a point at lower temperature where the melting line is regular (i.e., where it has a positive slope) and the HDW configuration is attained, for example, $330 \mathrm{~K} / 1.3 \mathrm{GPa}, D_{T}=$ $3.1 \times 10^{-5} \mathrm{~cm}^{2} / \mathrm{s}$ [28], we find that, within the uncertainty, they are identical. Our measurements thus indicate that the diffusion coefficient in water varies very little along the high temperature-high pressure melting line.

This observation might hold for other molecular liquids and provide a criterion for estimating diffusion coefficients along melting lines at extreme pressures and temperatures relevant for the physics of water-rich planets [37,38], where measurements are unfeasible, and extrapolations 
from moderate pressure measurements can lead to erroneous predictions.

We thank R. Auger and J. L. Laborier at Institut Laue Langevin for technical assistance. We are grateful to D. Chandler and R. Pick for fruitful discussions. This work was financially supported by the French Agence Nationale de la Recherche (ANR JCJC0135). This work is based on experiments performed at the Institut Laue-Langevin, Grenoble, France. This work is partially based on experiments performed at the Swiss spallation neutron source SINQ, Paul Scherrer Institute, Villigen, Switzerland. This work was performed using HPC resources from GENCI-IDRIS (Grant No. 2012-091387).

[1] Water: A Comprehensive Treatise, edited by F. Franks (Plenum, New York, 1972), Vol. 1, Chap. 12.

[2] Y. Katayama, T. Hattori, H. Saitoh, T. Ikeda, K. Aoki, H. Fukui, and K. Funakoshi, Phys. Rev. B 81, 014109 (2010).

[3] A. F. Goncharov, N. Goldman, L. E. Fried, J. C. Crowhurst, I. Feng, W. Kuo, C. J. Mundy, and J. M. Zaug, Phys. Rev. Lett. 94, 125508 (2005).

[4] A. K. Soper and M. A. Ricci, Phys. Rev. Lett. 84, 2881 (2000).

[5] A. M. Saitta and F. Datchi, Phys. Rev. E 67, 020201 (2003).

[6] T. Strässle, A. M. Saitta, Y. Le Godec, G. Hamel, S. Klotz, J. S. Loveday, and R. J. Nelmes, Phys. Rev. Lett. 96, 067801 (2006).

[7] T. Ikeda, Y. Katayama, H. Saitoh, and K. Aoki, J. Chem. Phys. 132, 121102 (2010);

[8] T. Kawamoto, S. Ochiai, and H. Kagi, J. Chem. Phys. 120, 5867 (2004).

[9] E. H. Abramson and J. M. Brown, Geochim. Cosmochim. Acta 68, 1827 (2004); E. H. Abramson, Phys. Rev. E 76, 051203 (2007).

[10] M. Krisch et al., Phys. Rev. Lett. 89, 125502 (2002).

[11] M. Santoro, F. A. Gorelli, T. Scopigno, M. Krisch, F. Sette, and G. Ruocco, Phys. Rev. B 84, 092301 (2011).

[12] Y. Takii, K. Koga, and H. Tanaka, J. Chem. Phys. 128, 204501 (2008); J. L. Aragones and C. Vega, J. Chem. Phys. 130, 244504 (2009).

[13] C. Cavazzoni, G. L. Chiarotti, S. Scandolo, E. Tosatti, M. Bernasconi, and M. Parrinello, Science 283, 44 (1999); E. Katoh, H. Yamawaki, H. Fujihisa, M. Sakashita, and K. Aoki, Science 295, 1264 (2002).

[14] A. Cunsolo, A. Orecchini, C. Petrillo, and F. Sacchetti, J. Chem. Phys. 124, 084503 (2006); 114, 16713 (2010).

[15] J. Jonas, T. DeFries, and D. J. Wilbur, J. Chem. Phys. 65, 582 (1976).
[16] J. Jonas, T. DeFries, and D. J. Wilbur, J. Chem. Phys. 65, 582 (1976).

[17] E. W. Lang and H. D. Lüdemann, Ber. Bunsenges. Phys. Chem. 85, 603 (1981); E. W. Lang and H. D. Lüdemann, Angew. Chem., Int. Ed. Engl. 21, 315 (1982).

[18] S. Klotz, T. Strässle, and L. E. Bove, Appl. Phys. Lett. (to be published); ILL Experimental Report No. 6-02-435; PSI Experimental Report No. 20080623.

[19] S. Klotz, in Techniques in High Pressure Neutron Scattering, (CRC press, Boca Raton, 2012).

[20] M. Bée, in Quasielastic Neutron Scattering (Taylor \& Francis, Bristol, 1988); V. F. Sears, Can. J. Phys. 45, 237 (1967).

[21] K. S. Singwi and A. Sjölander, Phys. Rev. 119, 863 (1960); C. T. Chudley and R. J. Elliott, Proc. Phys. Soc. London 77, 353 (1961).

[22] S. H. Chen, J. Teixeira, and R. Nicklow, Phys. Rev. A 26, 3477 (1982); J. Teixeira, M.C. Bellissent-Funel, S. H. Chen, and A. J. Dianoux, Phys. Rev. A 31, 1913 (1985).

[23] S. H. Chen, P. Gallo, F. Sciortino, and P. Tartaglia, Phys. Rev. E 56, 4231 (1997).

[24] J. Qvist, H. Schober, and B. Halle, J. Chem. Phys. 134, 144508 (2011).

[25] J. Qvist, E. P. Sunde, and B. Halle, J. Chem. Phys. 136, 204505 (2012).

[26] F. Volino and A. J. Dianoux, Mol. Phys. 36, 389 (1978).

[27] See Supplemental Material at http://link.aps.org/ supplemental/10.1103/PhysRevLett.111.185901 for high pressure neutron scattering apparatus and experiment details, data fitting model details, and molecular dynamics simulations details.

[28] K. Krynicki, C.D. Green, and D. W. Sawyer, Faraday Discuss. Chem. Soc. 66, 199 (1978).

[29] K. R. Harris and L. A. Woolfe, J. Chem. Soc., Faraday Trans. 1 76, 377 (1980)

[30] P. A. Egelstaff, in An Introduction to the Liquid State, edited by Clarendon (Oxford University Press, Oxford, 1992).

[31] S. R. Becker, P. H. Poole, and F. W. Starr, Phys. Rev. Lett. 97, 055901 (2006), and references therein.

[32] S. K. Kumar, G. Szamel, and J. F. Douglas, J. Chem. Phys. 124, 214501 (2006).

[33] F. Saija, A. M. Saitta, and P. V. Giaquinta, J. Chem. Phys. 119, 3587 (2003); R. Esposito, F. Saija, A. M. Saitta, and P. V. Giaquinta, Phys. Rev. E 73, 040502 (2006).

[34] R. Mills, J. Phys. Chem. 77, 685 (1973).

[35] R. Ludwig, F. Weinhold, and T. C. Farrar, J. Chem. Phys. 103, 6941 (1995).

[36] D. Laage, J. Phys. Chem. B 113, 2684 (2009); D. Laage and J. T. Hynes, J. Phys. Chem. B 112, 14230 (2008).

[37] H. Hussmann, F. Sohl, and T. Spohn, Icarus 185, 258 (2006).

[38] W. B. Hubbard, Science 275, 1279 (1997). 